\title{
Time to Step It Up: Mobile Health Intervention for Lifestyle Modification in Patients with Nonalcoholic Fatty Liver Disease
}

\author{
Alison Faust ${ }^{1,2}$. Jonathan G. Stine $\mathrm{e}^{1,2,3,4}$ \\ Accepted: 12 April 2021 / Published online: 3 May 2021 \\ (c) The Author(s), under exclusive licence to Springer Science+Business Media, LLC, part of Springer Nature 2021
}

Though there is no cure or effective drug therapy for nonalcoholic fatty liver disease (NAFLD), a leading cause of chronic liver disease worldwide, lifestyle modification with diet and regular physical activity remains the cornerstone of NAFLD treatment. Although there are weight lossindependent benefits of regular physical activity, including loss of liver fat and body fat, gains in physical fitness and improvement in endothelial dysfunction [1], lifestyle modification remains a vehicle to achieve modest weight loss with the ultimate goal of improving liver histology. The body of literature investigating lifestyle modification in patients with NAFLD is limited by strong heterogeneity, both in the population studied as well as the lifestyle intervention itself. Consequently, the optimal lifestyle modification in patients with NAFLD remains unknown. As NAFLD prevalence is expected to increase over $20 \%$ by 2030 [2], there is a clear, unmet need to determine the most effective lifestyle modification in order to lessen the large global public health burden this common disease presents.

In recognition of this highly significant knowledge gap, the American Gastroenterological Association (AGA) published a recent clinical practice update in an attempt to standardize best practices for lifestyle modification in all patients with NAFLD [3]. In order to achieve the desired weight loss goal of $5-10 \%$, the AGA guidance recommends creating

Jonathan G. Stine

jstine@pennstatehealth.psu.edu

1 Division of Gastroenterology and Hepatology, Department of Medicine, Pennsylvania State University Milton S.

Hershey Medical Center, 500 University Drive, Hershey, PA 17033, USA

2 Liver Center, The Pennsylvania State University- Milton S. Hershey Medical Center, Hershey, PA, USA

3 Department of Public Health Sciences, Pennsylvania State University Milton S. Hershey Medical Center, Hershey, PA, USA

4 Cancer Institute, Pennsylvania State University Milton S. Hershey Medical Center, Hershey, PA, USA a daily energy deficit of $500-1,000 \mathrm{kcal}$ so as to induce a reasonable rate of medically safe weight loss. This deficit is created by combining a hypocaloric diet (which can include the Mediterranean diet or a diet of similar design) that minimizes saturated fatty acids, red and processed meats, and high fructose corn syrup, with regular physical activity that aligns with physical activity guidelines for all adults from the Department of Health and Human Services. Ideally, 150-300 min of moderate-intensity or 75-150 min of vigorous-intensity activity should be completed each week.

Despite our longstanding understanding that lifestyle modification is important for overall and liver-specific health in patients with NAFLD, modest weight loss presents an onerous challenge to the clinician and patient alike. Even in a structured, highly supervised setting, weight loss goals are achieved by less than $10 \%$ of patients with NAFLD [4]. Moreover, $75 \%$ of patients with NAFLD will not achieve recommended amounts of physical activity despite almost all patients expressing desire to be more physically active [5].

What prevents patients with NAFLD from successfully attaining lifestyle modification? Common self-identified barriers include a lack of education and healthy lifestyle resources, physical discomfort, time and cost [5]. Mobile health (mHealth) applications offer the promise of removing many of these self-identified barriers, enabling the achievement of successful lifestyle modification. mHealth applications lead to higher quality clinical care, better patient-reported outcomes and improved self-management of chronic non-NAFLD metabolic disease, including obesity and pre-diabetes [6]. Whether or not mHealth is feasible, acceptable or effective remains an important knowledge gap in the care of patients with NAFLD.

In this issue of Digestive Diseases and Sciences, Tincopa et al. [7] endeavor to answer this question with their sixmonth pilot study in which they assessed a priori defined feasibility and acceptability of a mHealth-based lifestyle modification program in 40 patients with NAFLD. The 
authors also explored the clinical benefits of their mHealth intervention with their secondary clinical endpoints, including body weight, physical fitness (six-min walk test), hepatic transient elastography, routine laboratory testing, and health-related quality-of-life, with the goal of informing future large-scale trial design. The investigators adopted a previously validated protocol in non-NAFLD patients with metabolic disease in which they combined fitness activity trackers paired with a commercial mHealth fitness application with structured dietary feedback and education. Using these resources, the investigators used daily step counts to inform weekly or bi-weekly physical activity coaching with progressive step count goals. Dietary feedback and Mediterranean diet (or carbohydrate-controlled diet in participants with diabetes) based education were provided at baseline, month three and month six of program.

With these methods, Tincopa et al. demonstrated both acceptability (63\% enrollment rate) and feasibility (83\% 6-month completion rate). This was confirmed by qualitative interviewing techniques in which 59\% of participants reported the fitness activity tracker and mHealth application was easy to use and $66 \%$ identified step count feedback to be motivational in increasing their physical activity. Further confirming feasibility, fitness activity tracker compliance was excellent at $91 \%$. This is important because previous studies have found only $1 / 3$ of patients with NAFLD have used a fitness activity tracker in a real-world setting, supporting concerns about widespread use of this technology in this patient population [5].

Despite the promising feasibility and acceptability data, only $18 \%$ of NAFLD subjects achieved at least $5 \%$ weight loss, the minimal clinically important difference required to improve liver histology. Though this result is disappointing, it may be explained in part by the finding of minimal change in the median step count at the end of the intervention period, implying the study population continued to meet step count-based definitions of physical inactivity and that no large-scale activity changes occurred [8]. Given the success of fitness activity tracker use, oftentimes without mHealth application pairing, to increase daily step count in other physically inactive populations, including those with metabolic disease [9], and the fact that for each 1,000 step/d increase there is a 5\% reduction in overall mortality [10], this low-resource intervention should be effective in patients with NAFLD. Given the feasibility and acceptability data presented by Tincopa et al., the medical community awaits larger-scale studies designed to definitively address the value of fitness activity tracker use paired with mHealth application in increasing daily step count, preferably in parallel with modest weight loss. As the population in this feasibility study was very heterogenous, including $30 \%$ patients with cirrhosis, we suggest that more selective inclusion criteria in a proposed study would yield less ambiguous data. Such studies would be in contrast to current research study design that is focused on pharmacologic interventions in preference to lifestyle modifications in NAFLD patients with late-stage disease.

In terms of other clinical benefits, the investigators found statistically, but not clinically significant improvement in exploratory clinical outcomes, including six-min walk test, serum cholesterol (low-density and high-density lipoprotein), serum triglyceride level, and hemoglobin A1c. Without an improvement in daily step count with the intervention, additional investigation is necessary in order to explain these important observed clinical benefits. It is plausible that dietary intervention was the more beneficial aspect of this mHealth lifestyle modification program, as fidelity issues with accurate step count recording seem less likely based on the rigor with which the authors monitored physical activity.

Despite these limitations, the study is a nice addition to a growing body of literature that shows that mHealth-based lifestyle modification can help NAFLD patients achieve some degree of weight loss, since $45 \%$ of subjects did lose body weight. Whether or not a more intensive mHealthbased lifestyle modification would result in even greater weight loss remains unknown. Across the obesity medicine literature, five key components of a successful technologybased weight loss program have been identified: self-monitoring, counselor feedback, social support, structure and principles of behavior change, and individual tailoring of the program to the participant. Mobilizing these resources can require a large number of socioeconomic resources and "electronic device literacy," each of which may pose additional, novel barriers to enacting a more comprehensive mHealth-based lifestyle modification program in patients with NAFLD. Though Tincopa et al. did not find any issues with "electronic device literacy," their study cohort was highly educated; $65 \%$ of subjects had at least an undergraduate degree, which is more than two times greater than previous population-based reports of educational level in patients with NAFLD [11]. This difference in educational level biases towards "electronic device literacy"; whether or not a less educated population or a population with lower socioeconomic status would achieve similar "electronic device literacy" is unknown.

In summary, in the absence of a cure or regulatory agency-approved pharmacologic therapy for patients with NAFLD, lifestyle modification designed to achieve sustained, modest weight loss remains the most effective treatment for this leading cause of chronic liver disease. Despite the research to date and the longstanding clinical experience with recommending lifestyle modification, sustained weight loss remains hopelessly difficult to accomplish for many of our patients. As clinicians caring for patients with NAFLD, we now have data supporting the feasibility of mHealth application use to augment our current educational practices 
which we hope will achieve meaningful dietary change and regular physical activity. We look to future studies to definitively demonstrate the anticipated efficacy of mHealth-based lifestyle modification that has been observed in other chronic metabolic diseases.

\section{Declaration}

Conflict of interest The authors certify that they have no affiliations with or involvement in any organization or entity with any financial interest, or non-financial interest in the subject matter or materials discussed in this manuscript.

\section{References}

1. Thorp A, Stine JG. Exercise as medicine: the impact of exercise training on nonalcoholic fatty liver disease. Current Hepatology Rep. 2020;19:402-411.

2. Estes C, Razavi H, Loomba R, Younossi Z, Sanyal AJ. Modeling the epidemic of nonalcoholic fatty liver disease demonstrates an exponential increase in burden of disease. Hepatology. 2018;67:123-133.

3. Younossi ZM, Corey KE, Lim JK. AGA clinical practice update on lifestyle modification using diet and exercise to achieve weight loss in the management of nonalcoholic fatty liver disease: expert review. Gastroenterology. 2021;160:912-918.

4. Vilar-Gomez E, Martinez-Perez Y, Calzadilla-Bertot L et al. Weight loss through lifestyle modification significantly reduces features of nonalcoholic steatohepatitis. Gastroenterology. 2015; 149:367-378.

5. Stine JG, Soriano C, Schreibman Iet al. Breaking down barriers to physical activity in patients with nonalcoholic fatty liver disease Dig Dis Sci. 2020.

6. Toro-Ramos T, Michaelides A, Anton M et al. Mobile delivery of the diabetes prevention program in people with prediabetes: randomized controlled trial. JMIR mHealth and uHealth. 2020;8:e17842.

7. Tincopa M, Lyden, A, Wong, J, et al. Impact of a pilot structured mobile technology based lifestyle intervention for patients with non-alcoholic fatty liver disease. Dig Dis Sci. (Epub ahead of print). https://doi.org/10.1007/s10620-021-00603-w.

8. Tudor-Locke C, Bassett DR Jr. How many steps/day are enough? Preliminary pedometer indices for public health. Sports Med (Auckland, NZ). 2004;34:1-8.

9. Hasan H, Attlee A, Jan Bin J, Mohamed H, Aris N, Bin W, Muda WAM. Counting footsteps with a pedometer to improve HMW adiponectin and metabolic syndrome among young female adults in the United Arab Emirates. J Obesity. 2018; 1597840.

10. Hall KS, Hyde DR Bassett et al. Systematic review of the prospective association of daily step counts with risk of mortality, cardiovascular disease, and dysglycemia. Int J Behav Nutrition Phys Activity. 2020;17:78.

11. Gerber L, Otgonsuren M, Mishra A et al. Non-alcoholic fatty liver disease (NAFLD) is associated with low level of physical activity: a population-based study. Aliment Pharmacol Ther. 2012;36:772-781.

Publisher's Note Springer Nature remains neutral with regard to jurisdictional claims in published maps and institutional affiliations. 